\title{
Dengue, geoprocessamento e indicadores socioeconômicos e ambientais: um estudo de revisão
}

\author{
Regina Fernandes Flauzino, ${ }^{1}$ Reinaldo Souza-Santos ${ }^{2}$ \\ e Rosely Magalhães Oliveira ${ }^{3}$
}

Como citar Flauzino RF, Souza-Santos R, Oliveira RM. Dengue, geoprocessamento e indicadores socioeconômicos e ambientais: um estudo de revisão. Rev Panam Salud Publica. 2009;25(5):456-61.

RESUMO Objetivo. Analisar os estudos que abordaram o tema dengue e geoprocessamento juntamente com indicadores socioeconômicos e ambientais na busca de uma melhor compreensão do comportamento da doença.

Método. Conduziu-se uma busca nas bases MEDLINE, SciELO, Lilacs e banco de teses CAPES com os termos "dengue, sistema de informação geográfica, análise espacial, geoprocessamento, sensoriamento remoto e indicadores socioeconômicos e ambientais". Também foi conduzida uma busca manual de artigos selecionados das listas de referências. Foram incluídos todos os trabalhos publicados nos idiomas inglês, português ou espanhol até dezembro de 2007 que abordaram o tema dengue e geoprocessamento e indicadores socioeconomicos e ambientais. Os estudos foram agrupados conforme tipo (inquérito sorológico ou estudo com dados secundários) e unidade espacial de análise (município, distritos sanitários, bairros, regiões administrativas, setores censitários e quarteirões).

Resultados. Foram avaliados 22 estudos, todos da América Latina (19 do Brasil). Seis eram inquéritos sorológicos e 16 utilizaram dados secundários. Sistemas de informação geográfica foram utilizados em um inquérito e em 11 estudos da outra categoria. A agregação espacial utilizada foi semelhante em ambos os tipos. A pobreza não foi fator preponderante para o risco da doença. A heterogeneidade espacial de condições de vida e incidência esteve presente em 15 dos 16 trabalhos com dados secundários.

Conclusões. Como a complexidade da dengue está intimamente relacionada com as características ecológicas do ambiente, os estudos que utilizam agregados de unidades espaciais aliados à análise das características ambientais locais fornecem uma visão mais completa da doença e permitem a identificação de heterogeneidade espacial, que mostrou ser um aspecto importante para o entendimento do desenrolar da epidemia de dengue.

Palavras-chave Dengue/epidemiologia, indicadores ambientais, indicadores sociais, vigilância epidemiológica, sistema de informação geográfica.

Universidade Federal Fluminense (UFF), Departamento de Epidemiologia. Correspondência: Rua Professor Henrique Costa 296/204, Pechincha, CEP 22770-233, Rio de Janeiro, RJ, Brasil. Fone: +55-212629.9352/+55-21-2425.6191; e-mail: rflauzino@ uol. com.br

2 Fundação Oswaldo Cruz, Escola Nacional de Saúde Pública, Departamento de Endemias Samuel Pessoa. E-mail: rssantos@ensp.fiocruz.br

3 Fundação Oswaldo Cruz, Escola Nacional de Saúde Pública, Departamento de Endemias Samuel Pessoa. E-mail: rosely@ensp.fiocruz.br
A dengue é uma arbovirose que vem preocupando as autoridades sanitárias de todo o mundo em virtude de sua circulação nos cinco continentes e do grande potencial para o desenvolvimento de formas graves e letais de doença. Cerca de 2,5 bilhões de pessoas encontram-se em risco de infecção, particularmente em países tropicais, onde a umidade e a temperatura favorecem a proliferação do mosquito vetor, Aedes aegypti (1).

Entretanto, nem sempre os estudos que buscam associar a ocorrência da dengue com condições socioeconômicas e ambientais encontram resultados concordantes. Vários autores apontam uma relação proporcionalmente inversa entre a 
incidência da doença e as condições de vida (2-6). Em oposição, outros apontam uma relação positiva entre as condições de vida e a ocorrência da doença $(7,8)$. Esses autores sugerem que as diferenças entre as associações encontradas em diferentes pesquisas podem estar relacionadas ao tipo de unidade espacial utilizada (setor censitário, bairros, distritos e/ou municípios). Outra possibilidade seria uma limitação resultante da agregação espacial, já que, dependendo do nível de agregação, a captação da realidade pode não ser a ideal (9), ou seja: dependendo do modo como as variáveis foram agregadas para descrever grandes regiões, podem produzir grande variação de resultados. Esse problema pode ser agravado quando existem desigualdades socioeconômicas e infraestruturais. À medida que aumenta a agregação, a informação das covariáveis poderá discriminar menos as regiões e, consequentemente, os resultados poderão se tornar enganosos (10). Sendo assim, estudar o efeito de diferentes formas de agregação dos dados no tempo e no espaço pode ser relevante para os estudos da distribuição dos riscos da doença (11).

Nesse contexto, Silveira (12) encontrou "padrões complexos, que não corroboraram a expectativa baseada no senso comum, de uma relação linear entre dengue e pobreza em Niterói, Estado do Rio de Janeiro. Os resultados encontrados não pareceram consistentes com as hipóteses mais comumente levantadas para explicar o comportamento da dengue". Para o autor, "as características socioambientais particulares do município devem ser as mais relevantes para compreender o comportamento do processo endêmico-epidêmico da doença" (p. 73).

Nessa linha, sabe-se, atualmente, que uma das maneiras de se conhecer mais detalhadamente as condições de saúde da população é por intermédio de mapas que permitam observar a distribuição espacial de situações de risco e de problemas de saúde, ou seja, mediante a utilização de técnicas de geoprocessamento (13). A abordagem espacial permite a integração de dados demográficos, socioeconômicos e ambientais, promovendo o inter-relacionamento de informações advindas de diversos bancos de dados (14). A utilização de agregados espaciais, em especial com sistemas de informação geográfica (SIG), permite análises complexas, com rápida formação e alternação de cenários. Isso facilita a tomada de decisões e contribui para uma melhor compreensão dos problemas atuais de saúde $(13,15)$.

Assim, este trabalho teve como objetivo analisar os estudos que abordaram o tema dengue e geoprocessamento e indicadores socioeconômicos e ambientais na busca de uma melhor compreensão do comportamento da doença.

\section{MÉTODOS}

A busca bibliográfica foi conduzida nas bases MEDLINE (http://www.ncbi.nlm. nih.gov/pubmed), SciELO (http:/ / www. scielo.org), Lilacs (http://bases.bireme. br) e banco de teses CAPES (http:// www.capes.gov.br/servicos/banco-deteses) utilizando-se a combinação dos termos "dengue, sistema de informação geográfica, análise espacial, geoprocessamento, sensoriamento remoto, e indicadores socioeconômicos e ambientais" (em espanhol, dengue, sistema de información geográfica, análisis espacial, geoprocesamiento, teledetección, indicadores socio-ambientales; e em inglês, dengue, geographic information system, spatial analysis, geoprocessing, remote sensing, socio-environmental indicators).

Além disso, foi conduzida uma busca manual de publicações com base nas referências bibliográficas listadas nos artigos identificados.

Foram incluídos todos os trabalhos publicados nos idiomas inglês, portu- guês ou espanhol até dezembro de 2007 que abordaram o tema dengue e geoprocessamento e indicadores socioeconômicos e ambientais (figura 1). O processo de seleção dos estudos foi realizado por um dos autores (RFF). Para a extração dos dados, foi construído um formulário específico, onde foram registradas as informações concernentes a autor e ano, ano de desenvolvimento do estudo, idioma do país, cidade e área do estudo, tipo e periódico de publicação, objetivo do estudo, tipo de agregação espacial, indicadores socioeconômicos e atributos utilizados, tipos de software utilizados, resultados e limitações.

Os trabalhos selecionados foram divididos nas categorias inquéritos sorológicos e estudos que utilizavam dados secundários dos órgãos oficiais de saúde. Tal divisão pretendeu buscar diferenças no padrão de análise entre estudos baseados em dados primários ou secundários. Alguns autores declaram preferência por estudos baseados em inquéritos sorológicos, uma vez que eles tendem a contribuir para a diminuição de viés associado aos dados de origem secundária (16).

As unidades espaciais de análise foram classificadas nos seguintes níveis: município, distritos sanitários, bairros, regiões administrativas, setores censitários e quarteirões. Agregado espacial, neste estudo, equivale a agregações dessas unidades espaciais.

FIGURA 1. Fluxograma da seleção de estudos para inclusão no artigo de revisão sobre dengue, geoprocessamento e indicadores socioeconômicos e ambientais

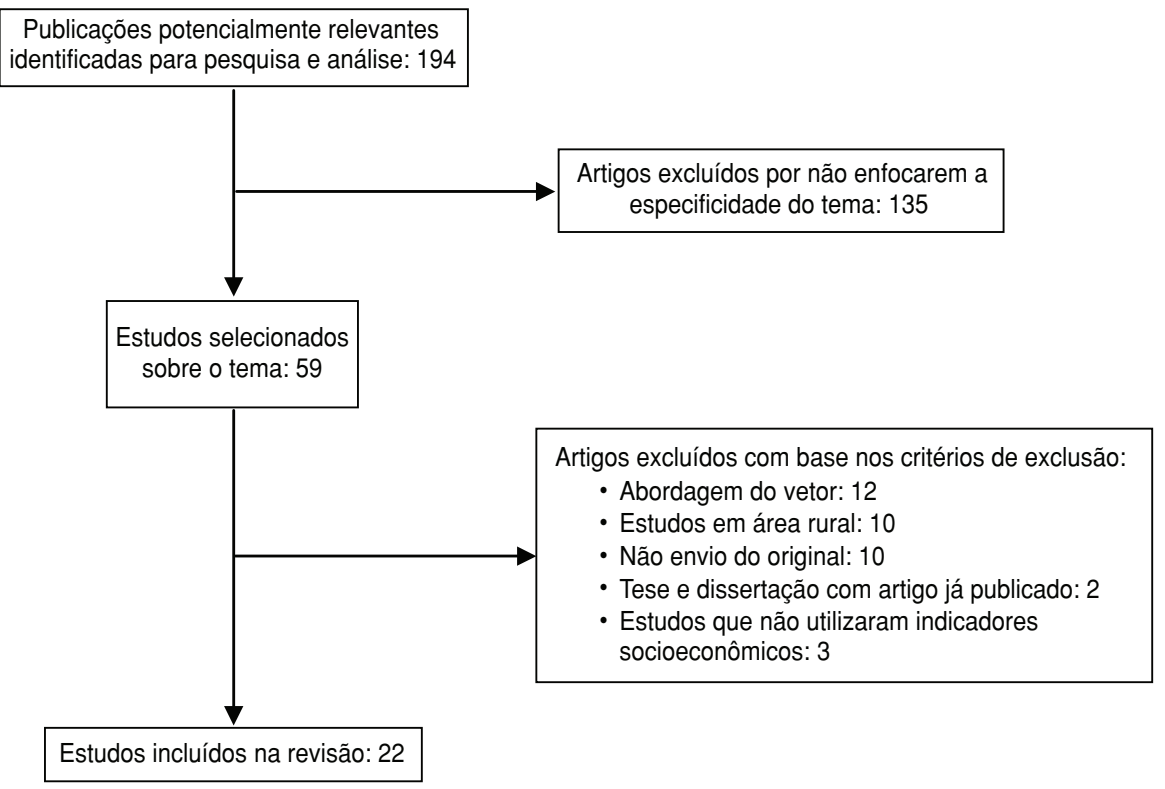


Os indicadores socioeconômicos utilizados foram os preconizados pela Rede Interagencial de Informações para a Saúde (RIPSA) (17). Os indicadores ambientais foram relacionados à drenagem urbana, limpeza pública e pontos estratégicos para vigilância de Aedes aegypti, de acordo com o preconizado pela Fundação Nacional de Saúde (FUNASA) (18). A utilização desses parâmetros teve como finalidade obter um maior nível de comparabilidade entre os estudos analisados.

Foram identificados trabalhos que fazem associação entre dengue e heterogeneidade espacial e, por isso, essa categoria foi incluída. A heterogeneidade espacial foi definida neste estudo como um espaço geográfico onde se encontram populações em diferentes estratos socioeconômicos. Esse espaço pode ser um agregado de unidades espaciais como setores censitários, bairros e/ou distritos sanitários. Por sua vez, a heterogeneidade de incidência e/ou de distribuição de casos está relacionada às diferentes condições de vida dos diferentes estratos sociais que ocupam o espaço. A maneira como os espaços são ocupados por populações de diferentes estratos socioeconômicos pode tornar tais espaços vulneráveis e criar condições que favoreçam a produção e a reprodução de doenças $(19,20)$. Assim, no caso da dengue, quando nos referimos à heterogeneidade espacial de distribuição de casos e/ou incidência, estamos nos referindo também à heterogeneidade espacial de condições de vida. Cada localidade possui uma historicidade própria, fruto de processos sociais e políticos singulares, podendo ser, nesse nível, evidenciada a particularidade dos processos de transmissão da doença em que a produção e reprodução se concretizam (19).

\section{RESULTADOS}

A busca resultou em 22 trabalhos que atendiam os critérios utilizados. Em relação ao país de origem, $100 \%$ foram produzidos na América Latina, sendo 19 no Brasil (5 dissertações de mestrado, 1 tese de doutorado e 13 artigos), um na Argentina, um na Venezuela e um em Cuba. Os estudos produzidos em outros continentes, especialmente na Ásia, versavam sobre o vetor ou estudavam variáveis relacionadas ao ambiente rural, por isso foram excluídos deste estudo.

Os artigos selecionados foram publicados em inglês, português ou espanhol.
Seis eram inquéritos sorológicos e 16 utilizaram dados secundários. Dos inquéritos sorológicos, somente um utilizou SIG; dos estudos com dados secundários, 11 utilizaram SIG.

\section{Inquéritos sorológicos}

As variáveis "presença prévia da doença" e "vacina contra febre amarela" estiveram presentes em todos os artigos selecionados. Dentre eles, somente um (21) usou SIG. Os indicadores utilizados em todos os estudos foram: sexo, idade, densidade populacional, situação/estado civil e condições de moradia (indicadores demográficos); instrução e renda (socioeconômicos); instalação sanitária de água e esgoto, lixo (de cobertura se serviços); e presença de inservíveis, ou seja, detritos que não podem ser reutilizados (ambientais).

Em relação ao nível de agregação, foi identificado apenas um estudo pautado em cada uma das unidades espaciais descritas a seguir: município (22), distrito sanitário (7) e bairro (23). Três estudos utilizaram como unidade de análise os setores censitários $(16,21,24)$.

No que tange aos fatores de risco, nos estudos cujas unidades eram municípios e distritos sanitários, foi identificado risco na população de maior renda (7, 22). Contudo, esses estudos não apresentaram diferença estatística para risco em relação ao sexo, faixa etária e escolaridade. A presença de heterogeneidade espacial na distribuição de casos foi detectada no estudo de Vasconcellos et al. (7), que utilizaram como unidade os distritos sanitários. Em relação ao estudo com bairros (23), foi identificado risco em mulheres, não havendo diferenciação por faixa etária. Já os estudos com base em setores censitários $(21,24,25)$ identificaram risco nas faixas etárias mais elevadas e correlação positiva entre soropositividade e escolaridade. Contudo, não foi identificada diferença de risco entre as diferentes condições de vida (aspectos socioeconômicos). O estudo que utilizou um indicador ambiental (24) identificou risco em setores censitários que apresentaram presença de inservíveis.

\section{Dados secundários}

Dos 16 estudos com dados secundários pautados pela agregação espacial, 13 foram produzidos no Brasil $(2,8-12,26-$ 32), um na Venezuela (33), um em Cuba
(34) e um na Argentina (35). Os indicadores utilizados nos estudos com dados secundários foram: sexo, idade, densidade demográfica, situação civil, razão de dependência (mede a participação relativa da população potencialmente inativa, que deveria ser sustentado pela parcela da população produtiva), razão de sexos (número de homens para $100 \mathrm{mu}$ lheres) e condições de moradia (demográficos); instrução, renda, índice de pobreza e favelas (socioeconômicos); instalação sanitária de água e esgoto, coleta de lixo (de cobertura de serviços); índice de infestação e criadouros do vetor, borracharias, cemitérios, ferro-velho (ambientais e operacionais); índice pluviométrico, umidade relativa e temperatura (climáticos/meteorológicos); e ano, mês e estações climáticas no momento do estudo (temporais).

Em relação ao nível de agregação, o estudo que utilizou o município como unidade de análise identificou como fatores de risco a faixa etária de 15 a 49 anos e a presença de criadouros; não encontrou, todavia, diferença entre os sexos (26).

Dos estudos que utilizaram bairros como unidade de análise $(9,10,12,25$, $27,28,33)$, três $(9,27,28)$ identificaram o sexo feminino como mais exposto ao risco de dengue. Os demais (10, 12, 25, 33) não encontraram diferença de risco entre os sexos. A faixa etária na qual se identificou maior risco foi aquela, segundo Machado (27), considerada economicamente ativa, que variou de 20 a 29 anos no trabalho de Santos (25) até 20 a 39 anos no trabalho de Ribeiro et al. (28). Risco para dengue foi também identificado em áreas de maior concentração de indivíduos, grande diversidade econômica, forte adensamento populacional, alta densidade domiciliar, alta densidade de lotes vagos (terrenos baldios), grande via de circulação de mercadorias e pessoas, falta de oferta de emprego e áreas com deficiência no sistema de coleta de esgoto $(25,33)$.

A heterogeneidade espacial de incidência de casos só não foi identificada em um estudo (28). Considerando o uso de SIG, todos os quatro estudos que utilizaram essa ferramenta identificaram heterogeneidade espacial $(10,12,27,33)$. Ainda em relação aos estudos com bairros e SIG, o estudo de Ferreira (10) demonstrou que a presença de criadouros (borracharias, cemitérios, ferro-velho, terreno baldio e caixas d'água descobertas) foi um fator de risco para a doença. 
Ressalta-se que, nos estudos por bairros, a pobreza não foi fator preponderante para risco da doença. O estudo de Medronho (29) utilizou regiões administrativas como unidade espacial e identificou risco de ocorrência de dengue nas regiões onde havia maior percentual de população favelada, maior densidade demográfica, menor cobertura de água encanada, de instalação sanitária própria e de iluminação elétrica. O desfecho difere dos demais estudos ao concluir que a diminuição do risco está associada à melhora dos parâmetros econômicos da população e que existe homogeneidade espacial na distribuição da incidência dos casos.

Os fatores de risco identificados pelo estudo que utilizou distritos sanitários como unidade espacial (30) foram: a faixa etária elevada, a presença de áreas densamente povoadas e a maior proporção de população carente; porém o último fator, de acordo com Teixeira (16), pode ser reflexo de viés de notificação, visto que essa população utiliza em maior volume os serviços públicos de saúde, que são a principal fonte de notificação de doenças e agravos à saúde.

Em relação aos estudos baseados em setores censitários, Paulino e Natal (2) utilizaram a estratificação de setores censitários como método de análise com a finalidade de caracterizar aglomerados de setores com características socioambientais possivelmente homogêneas. Dos quatro estudos, todos associaram o risco de contrair dengue aos setores com maior renda, e três $(31,32,36)$ identificaram heterogeneidade espacial da distribuição de casos. Esses três estudos utilizaram SIG.

Martinez et al. (34) e Bottinelli et al. (35) utilizaram a metodologia de estratificação de quarteirões, buscando identificar agregados com características socioambientais relativamente homogêneas, estratificação espacial da vulnerabilidade à dengue e identificação de áreas de risco. Em ambos foram utilizados SIG.

$\mathrm{Na}$ análise para fatores de risco, ficou evidente que os agregados de quarteirões que apresentaram alta densidade populacional, presença de grandes centros comerciais e educacionais e presença de vetor foram os de maior risco para a doença. As áreas com presença de alojamentos para turistas e hotéis e com presença do vetor também foram consideradas de risco (34). De forma semelhante, Bottinelli et al. (35) identificaram áreas de risco em agregados de quarteirões onde havia forte intercâmbio de pessoas e presença de vetor. A heterogeneidade espacial de casos foi identificada nesses dois estudos. A elevada diferenciação entre os quarteirões sugere diferenças na vulnerabilidade espacial para a introdução e a transmissão da dengue, e a elevada associação estatística com a espacialidade dos casos notificados valida os resultados da estratificação espacial da vulnerabilidade da dengue (34).

\section{Foco na unidades espaciais: municípios}

Tanto o estudo pautado em dados secundários (26) quanto o soroepidemiológico (22) apresentaram resultados semelhantes ao evidenciar maior risco nas faixas etárias mais elevadas e não registrar diferenças entre os sexos. Torres (37) comenta que as populações afetadas pela dengue em localidades antes indenes são aquelas de faixas etárias mais elevadas, por serem mais suscetíveis, e as mulheres, por conta da característica domiciliar do vetor. No entanto, apesar de ambos os estudos terem sido conduzidos em anos de epidemias recentes, a última característica não foi observada nos estudos que utilizaram a agregação por município $(22,26)$.

O estudo de dados secundários que utilizou variáveis ambientais (26) revelou associação entre dengue e presença de criadouros na localidade.

\section{Distritos sanitários}

A heterogeneidade espacial da distribuição da doença foi ressaltada nos dois tipos de estudo que utilizaram a agregação por distritos sanitários $(7,30)$; divergem, entretanto, quanto aos indicadores socioeconômicos e demográficos de risco.

Os resultados apresentados por Vasconcellos et al. (7), que se basearam em dados primários, apontam para uma relação de maior risco em áreas de maior poder aquisitivo. Já o estudo de dados secundários (30) aponta o risco em áreas de grande diversidade econômica e populacional, podendo este fato estar diretamente relacionado à heterogeneidade espacial da distribuição da doença.

Não foram observadas diferenças significativas na incidência de dengue em relação às variáveis sexo, idade e escolaridade nos dois tipos de estudo. Os níveis de exposição dos diferentes segmentos da população foram semelhantes, reforçando a tese de que a infecção não tem lugar particular para ocorrer, podendo se dar tanto nos domicílios como nas escolas ou locais de trabalho (7).

\section{Bairros}

A predominância do sexo feminino foi relatada tanto nos inquéritos sorológicos (23) quanto nos estudos com dados secundários $(9,27,28)$. Em relação à idade, no estudo sorológico (24) todas as faixas foram atingidas; porém, os resultados dos estudos com dados secundários apontam para maior risco em faixas etárias economicamente ativas $(9,10,12,25$, $27,28,33)$.

Sistemas de informações geográficas foram utilizados em quatro estudos com dados secundários (10, 27, 28, 33). Em todos a heterogeneidade espacial foi presença marcante, demonstrando que a distribuição da incidência da doença não foi uniforme no espaço. Os resultados desses estudos apontam também para o maior risco em áreas de melhores condições de vida, não sendo a pobreza um fator preponderante para o aumento ou diminuição do risco de adquirir dengue.

Em relação aos indicadores ambientais e de cobertura de serviços, a presença de criadouros e de água não proveniente de rede geral de abastecimento são apontados como fatores de risco para a doença $(10,25)$.

\section{Setores censitários}

Neste nível de agregação espacial, a heterogeneidade espacial também se fez presente tanto em inquéritos como em estudo com dados secundários. Os resultados se diferenciam, contudo, em termos dos indicadores socioeconômicos e de cobertura de serviços.

Nos estudos com dados primários (16, 24) não foi encontrada diferença de risco entre as condições de vida e os espaços ocupados; observou-se, todavia, maior risco para as faixas etárias mais elevadas e as com maior escolaridade.

O estudo de Barcellos et al. (36) mostrou que a possibilidade de aquisição de dengue era maior nas áreas de população de maior renda. Contudo, esse estudo analisou dados de casos importados, pois a área, até o momento do estudo, não tinha casos autóctones. Os estudos que estratificaram os setores com 
diferentes riscos $(2,32)$ encontraram resultados semelhantes: nível de incidência inversamente proporcional às condições de vida. As áreas de maior risco foram consideradas os agregados de setores censitários bem adensados, que possuíam população de menor renda e escolaridade, habitações simples e com carência de serviços básicos. Importante ressaltar que tais estudos foram realizados durante ou após a ocorrência da primeira epidemia de dengue nessas localidades.

\section{Quarteirões}

Neste nível de agregação foram selecionados somente dois estudos com dados secundários $(33,34)$. A estratificação de quarteirões com a finalidade de representar espacialmente a vulnerabilidade à dengue e identificar áreas de risco foi testada, sendo observada heterogeneidade espacial de casos com a utilização de SIG. Os fatores de risco foram alta densidade populacional, presença de grandes centros comerciais e educacionais e presença de vetor. As áreas de agregados de quarteirões com presença de vetor concomitantemente a alojamento para turistas e hotéis (34) e em áreas com forte intercâmbio de pessoas (35) foram consideradas de risco. Os autores validam a utilização de agregados de quarteirões ao afirmar que a estratégia permite estratificar espacialmente a vulnerabilidade da dengue por meio da identificação de áreas com diferentes associações estatísticas dos casos notificados.

\section{DISCUSSÃO}

Como mostrou a presente análise, os inquéritos e estudos com dados secundários que utilizaram o mesmo tipo de agregação espacial apresentaram resultados semelhantes. A heterogeneidade foi identificada em cerca de $80 \%$ dos trabalhos, sendo mais frequente em estudos realizados em regiões que sofreram mais de uma epidemia da doença. Além disso, essa distribuição espacial da doença ficou evidente em estudos que utilizaram SIG e níveis de agregação espacial de maior escala, tais como bairros, setores censitários e quarteirões. De acordo com Favier et al. (38), espera-se que, no futuro, os estudos levem em consideração o grau de heterogeneidade existente, que pode afetar epidemias como as de dengue. Além disso, estudos pautados em SIG podem permitir a análise em nível regional a partir de dados coletados em escalas locais.

Finalmente, é importante ressaltar que os estudos com abordagens de localiza- ções espaciais e uso de SIG, embora recentes na área de saúde, tornam-se aos poucos imprescindíveis para a análise da determinação das doenças. Esse tipo de estudo permite um importante resgate do papel da atmosfera socioambiental na produção e reprodução da doença e também, em última análise, mediante seus resultados, permite capacitar os serviços de saúde para o controle e a vigilância da doença (39). Além disso, constatamos que, no caso da dengue, foi importante o foco dos estudos com dados secundários tanto na agregação de unidades espaciais quanto nas características ambientais, que são aspectos típicos de estudos ecológicos (39) - diferenciando-se dos inquéritos sorológicos, que abordaram especialmente as características dos indivíduos, sem preocupar-se com o nível ambiental. Como a complexidade da dengue está intimamente relacionada com as características ecológicas do ambiente (além das características do indivíduo), os estudos que utilizam agregados de unidades espaciais aliados à análise das características ambientais locais permitem uma análise mais completa da doença e a identificação de heterogeneidade espacial nas localidades estudadas.

\section{REFERÊNCIAS}

1. Tauil PL. Aspectos críticos do controle do dengue no Brasil. Cad Saude Publica. 2002; 18(3):867-71.

2. Paulino AIC, Natal D. Distribuição espacial da dengue e determinantes sócio-econômicos em uma localidade urbana no sudeste do Brasil. Rev Saude Publica. 1998;32(3):232-36.

3. Vasconcelos PFC, Mota K, Straatmann A, Santos-Torres S, Rosa APAT, Tavares Neto J. Epidemia de dengue em Ipupiara e Prado, Bahia. Inquérito soro-epidemiológico. Rev Soc Bras Med Trop. 2000;33(1):61-7.

4. Costa MAR. A ocorrência do Aedes aegypti na região noroeste do Paraná: um estudo sobre a epidemia da dengue em Paranavaí - 1999, na perspectiva da geografia médica [dissertação]. São Paulo: Universidade Estadual Paulista Júlio de Mesquita/Presidente Prudente; 2004.

5. Maciel IJ. Avaliação epidemiológica do dengue no município de Goiânia no período de 1994 a 1997 [dissertação]. Goiânia: Instituto de Medicina Tropical, Universidade Federal de Goiás; 1998.

6. Siqueira Junior, JB. Vigilância do dengue: aplicação de diagramas de controle e análise espacial no município de Goiânia - Goiás [dissertação]. Goiânia: Instituto de Medicina Tropical, Universidade Federal de Goiás; 2001.

7. Vasconcelos PFC, Lima JWO, Rosa APAT, Timbó MJ, Rosa EST, Lima HR, et al. Epidemia de dengue em Fortaleza, Ceará: inquérito soro-epidemiológico aleatório. Rev Saude Publica. 1998;32(5):447-54.

8. Barcellos C, Pustai AK, Weber MA, Brito MRV. Identificação de locais com potencial de transmissão de dengue em Porto Alegre através de técnicas de geoprocessamento. Rev Soc Bras Med Trop. 2005;38(3):246-50.

9. Alcoforado VM. Dengue 2001-2002, município de Niterói: um estudo ecológico utilizando modelos hierárquicos bayesianos [dissertação]. Rio de Janeiro: Núcleo de Estudos em Saúde Coletiva, Universidade Federal do Rio de Janeiro; 2005.

10. Ferreira GS. Análise espaço-temporal da distribuição dos casos de dengue na cidade do Rio de Janeiro no período de 1986 a 2002 [dissertação]. Rio de Janeiro: Universidade Federal do Rio de Janeiro; 2004.

11. Almeida MCM. Distribuição espacial de casos notificados de dengue em Belo Horizonte, Minas Gerais, 1996-2002: Identificação de conglomerados e fatores de risco associados [dissertação]. Minas Gerais: Universidade Federal de Minas Gerais; 2004.

12. Silveira NAP. Distribuição territorial de dengue no município de Niterói, 1996 a 2003 [dissertação]. Rio de Janeiro: Escola Nacional de Saúde Pública, Fundação Oswaldo Cruz; 2005.

13. Lagrotta MTF. Geoprocessamento de indicadores entomológicos na identificação de áreas imóveis e recipientes chaves no controle do Aedes aegypti [dissertação]. Rio de Janeiro: Escola Nacional de Saúde Pública, Fundação Oswaldo Cruz; 2006.

14. Carvalho MS, Pina MF, Santos S. Conceitos básicos de sistemas de informação geográfica e cartografia aplicados à saúde. Brasília: Organização Pan-Americana da Saúde/Ministério da Saúde; 2000.

15. Barcellos C, Santos SM. Colocando dados no mapa: A escolha da unidade de agregação e integração de bases de dados em saúde e ambiente através do geoprocessamento. IESUS. 1997;6:21-9.

16. Teixeira MG. Dinâmica de circulação do vírus do dengue em um complexo centro urbano [tese]. Salvador: Instituto de Saúde da Comunidade, UFBA; 2000. 
17. RIPSA - Rede Interagencial de Informações para a Saúde. Indicadores básicos de saúde no Brasil: conceitos e aplicações. Brasília: Organização Pan-Americana da Saúde; 2002.

18. Fundação Nacional de Saúde. Dengue, instruções para pessoal de combate ao vetor: Manual de normas técnicas. $3^{\text {a }}$ ed. Brasília: Ministério da Saúde, FUNASA; 2001.

19. Sabroza PC, Toledo, LM, Osanai CH. A organização do espaço e processos endêmicosepidêmicos. Em: Leal MC, Sabroza PC, Rodrigues RH, Bus PM, eds. Saúde, ambiente e desenvolvimento. Vol II. São Paulo/Rio de Janeiro: Hucitec/Abrasco; 1992. Pp. 57-77.

20. Bernard P, Charafeddine R, Frohlich KL, Daniel M, Kestens Y, Potvin L. Health inequalities and place: A theoretical conception of neighbourhood. Soc Sci Med. 2007;65(9):1839-52.

21. Siqueira Júnior JB, Martelli CMT, Maciel IJM, Oliveira RMO, Ribeiro MGR, Amorm FP, et al. Household survey of dengue infection in Central Brazil: Spatial point pattern analysis and risk factors assessment. Am J Trop Med Hyg. 2004;71(5):646-51.

22. Vasconcellos PFC, Lima JWO, Raposo ML, Rodrigues SG, Travassos da Rosa JFS, Amorim SMC, et al. Inquérito soro-epidemiológico na Ilha de São Luis durante epidemia de dengue no Maranhão. Rev Soc Bras Med Trop. 1999;32(2):171-9.

23. Vasconcelos PFC, Travassos da Rosa ES, Freitas RB, Degallier N, Rodrigues SC, Travassos da Rosa APA. Epidemia de febre clássica de dengue causada pelo tipo 2 em Araguaína, Tocantins, Brasil. Rev Inst Med Trop São Paulo 1993;35(2):145-8.

24. Heukelbach J, Oliveira FAZ, Kerr-Pontesand LRS, Feldmeier H. Risk factors associated with an outbreak of dengue fever in a favela in Fortaleza, north-east Brazil. Trop Med Int Health. 2001;6(8):635-42.

25. Santos A. Geografia da dengue em Uberlândia (MG) na epidemia de 1999. Caminhos de Geografia. 2004;3(11)35-52.

26. Gonçalves Neto VS, Rebelo JM. Aspectos epidemiológicos do dengue no Município de São Luís, Maranhão, Brasil, 1997-2002. Cad Saude Publica. 2004;20(5):1427-31.

27. Machado JP. Dengue e condições de vida no município de Nova Iguaçu: uma abordagem espacial [dissertação]. Rio de Janeiro: Escola Nacional de Saúde Pública, Fundação Oswaldo Cruz; 2007.

28. Ribeiro AF, Marquesi GRAM, Voltolini JC Condino MLF. Associação entre incidência de dengue e variáveis climáticas. Rev Saude Publica. 2006;40(4):671-6.

29. Medronho R. A geografia da dengue no $\mathrm{Mu}-$ nicípio do Rio de Janeiro: uma análise do geoprocessamento [dissertação]. Rio de Janeiro: Escola Nacional de Saúde Pública, Fundação Oswaldo Cruz; 1993.

30. Teixeira MG. Epidemiologia da dengue em Salvador-Bahia, 1995-1999. Rev Soc Bras Med Trop. 2001;34(3):269-74.

31. Mondini A, Chiaravalloti Neto F, Sanches MG, Lopes JCC. Análise espacial da transmissão de dengue em uma cidade de porte médio do interior paulista. Rev Saude $\mathrm{Pu}$ blica. 2005;39(3):444-51.

32. Almeida MCM, Caiaffa WT, Assunção RM, Proietti FA. Spatial vulnerability to dengue in a Brazilian urban area during a 7-year surveillance. J Urban Health. 2007;84(3):334-45.

33. Barrera R, Delgado N, Jiménez M, Villalobos I, Romero Y. Estratificación de una ciudad hiperendémica en dengue hemorrágico. Rev Panam Salud Publica. 2000;8(4):225-33.
34. Martinez TTP, Rojas LI, Valdes LS, Remond $R$. Vulnerabilidad espacial al dengue. Una aplicación de los sistemas de información geográfica en el municipio Playa de Ciudad de La Habana. Rev Cubana Salud Publica. 2003; 29(4):353-65

35. Bottinelli OR, Marder G, Ulón SN, Ramírez L, Sario, HR. Estratificación de áreas de riesgodengue en la ciudad de Corrientes mediante el uso de los (SIG) sistemas de información geográfico. Corrientes: UNNE; 2002. Disponível em: www.unne.edu.ar/Web/cyt/cyt/ 2002/04-Veterinarias/V-062.pdf. Acessado em abril de 2009.

36. Barcellos C, Pusta AK, Weber MA, Brito MRV. Identificação de locais com potencial de transmissão de dengue em Porto Alegre através de técnicas de geoprocessamento. Rev Soc Bras Med Trop. 2005;38(3):246-50.

37. Torres EM. Dengue. Rio de Janeiro: Editora Fiocruz; 2005.

38. Favier C, Schmit D, Muller-Graf CDM, Cazelles B, Degallier N, Mondet B, et al. Influence of spatial heterogeneity on an emerging infectious disease: the case of dengue epidemics. Proc Biol Sci. 2005;272(1568):1171-7.

39. Souza-Santos R, Carvalho MS. Análise da distribuição espacial de larvas de Aedes aegypti na Ilha do Governador, Rio de Janeiro, Brasil. Cad Saude Publica. 2000;16(1):31-42.

Manuscrito recebido em 16 de julho de 2008. Aceito em versão revisada em 17 de novembro de 2008
ABSTRACT

\section{Dengue, geoprocessing, and socioeconomic and environmental indicators: a review}

\footnotetext{
Key words Dengue, epidemiology; environmental indicators, social indicators, epidemiologic

surveillance, geographic information system.
} on dengue and geoprocessing, as well as socioeconomic and environmental indicators. Method. MEDLINE, SciELO, and Lilacs databases, as well as the CAPES dissertation databank, were searched using the following key words: dengue, geographic information system, spatial analysis, geoprocessing, remote sensing, and socioenvironmental indicators. A manual search of the bibliographies of select articles was also performed. All studies published in English, Portuguese, or Spanish, through December 2007, that focused on dengue, geoprocessing, and socioeconomic and environmental indicators were included. The relevant articles were grouped according to type (serologic surveys or secondary data analyses) and spatial analysis unit (municipality, health district, neighborhood, administrative region, census tracts, and city blocks).

Results. Twenty-two studies from Latin America (19 from Brazil) were evaluated. Six were serologic surveys and 16 employed secondary data. Geographic information systems were employed in one survey, and 11 used secondary data analyses. Spatial clustering was similar in both types of studies. Poverty was not a major risk factor for the disease. Spatial heterogeneity of living conditions and incidence was reported by 15 of 16 studies with secondary data.

Conclusions. Since the complexity of dengue is closely tied to the ecological characteristics of the environment, studies based on spatial clusters plus local environmental determinants provide a more comprehensive view of the disease. These studies also allow for the identification of spatial heterogeneity, shown to be a key to understanding how dengue epidemics develop. 